\title{
La ciencia soviética migra al 'sur': Los científicos de la ex URSS en México y en Brasil
}

\author{
Soviet Science migrates 'South': Scientists from the Former USSR in Mexico and \\ Brazil
}

Isabel Izquierdo ${ }^{1}$

Artigo recebido em para publicação em jun./2014 e aceito para publicação em out./2014

\section{RESUMEN}

El objetivo del estudio fue conocer las experiencias de migración-inmigración de científicos de la ex URSS en Latinoamérica. Se aborda su inserción a los nuevos espacios académicos y se indaga, de manera exploratoria, la construcción de redes de investigación desde México y Brasil. Para acceder a las experiencias de los científicos, se utilizó el enfoque biográfico, a través del relato de vida. Los resultados que se obtuvieron fue que los itinerarios que han recorrido los científicos para llegar a ambos países fueron diferentes y también las formas en las que se incorporaron a los espacios académicos laborales. Mientras que en México se implementó un programa específico de atracción de científicos extranjeros, en Brasil, la llegada de éstos fue a través de vínculos con colegas. Con respecto a la exploración sobre su trabajo académico, se encontró que los científicos, tanto en México como en Brasil, en especial en el primer caso, no suelen mantener vínculos con sus colegas connacionales que viven en sus países de origen y en el caso de los que se encuentran en el segundo país, ese nexo se ha debilitado.

Palabras clave: Científicos. Ex URSS. Experiencias. Migración. Inmigración. México. Brasil.

\section{RESUMO}

O objetivo foi estudar as experiências de migração-imigração de cientistas da ex-União Soviética na América Latina. É estudado sua incorporação a novos espaços acadêmicos e explora, de maneira exploratória, a construção de redes de pesquisa do México e do Brasil. Para acessar as experiências dos cientistas, a abordagem biográfica foi utilizado através da história de vida. Os resultados obtidos foram que os caminhos que os cientistas tomaram para chegar aos dois países eram diferentes e também as formas em que eles chegaram os espaços académicos. Enquanto no México foi implementado um programa específico atrair cientistas estrangeiros, no Brasil sua chegada foi através de ligações com os colegas. Respeito à exploração de seu trabalho académico, se descobriu que tanto o México eo Brasil, especialmente no primeiro caso, geralmente não manter vínculos com seus compatriotas que vivem em seus países de origem e, no caso de aqueles encontrados no segundo país, esta ligação se enfraqueceu.

Palavras-chave: Cientistas. Ex-URSS. Experiências. Migração. Imigração. México. Brasil.

\section{ABSTRACT}

The aim of this study was to know the experiences of migration-immigration of scientists from the Former Soviet Union in Latin America. It shows their incorporation to new academic spaces and explores the building research networks from Mexico and Brazil. To access the experiences of scientists, the biographical approach was used through the life story. The results obtained were that the trails that scientists have followed to get to the two countries were different and the ways in which they joined the academic spaces too. While in Mexico a specific attraction program of foreign

\footnotetext{
${ }^{1}$ Doctora en Pedagogía por la Universidad Nacional Autónoma de México (UNAM). Fundadora de la Red Académica en Migración y Educación y coordinadora de su seminario en la Facultad de Filosofía y Letras, Posgrado en Pedagogía, de la misma universidad. Actualmente realiza una estancia posdoctoral en la Universidad Autónoma Metropolitana (UAM). E-mail: izcam@hotmail.com.
} 
scientists was implemented, in Brazil, scientists arrived through links with their colleagues. About the exploration of their academic work, scientists in both cases, especially in the first case, they do not usually maintain links with their countrymen colleagues that are living in their countries of origin, and in the second country, that kind of link has weakened.

Keywords: Scientists. Former USSR. Experience. Migration. Immigration. Mexico. Brazil.

\section{INTRODUÇÃO}

Latinoamérica es una región que tiene una vasta historia de exilios y de emigraciones. En ella, se han forzado y gestado desplazamientos, tanto por motivos políticos, sociales como económicos. Actualmente México, Cuba, Jamaica, Colombia, Brasil y Perú por mencionar algunos, integran el grupo de países con mayor emigración calificada (LOZANO Y GANDINI, 2010). Son estos mismos países, en especial dos de ellos, quienes también atraen hacia sus espacios de investigación y docencia a profesionales extranjeros para ocupar plazas de tiempo completo como académicos en universidades públicas. En la década de los noventa y dos mil, llamó la atención los esfuerzos por incorporar a científicos provenientes de la desaparecida Unión Soviética en países como México y Brasil ¿Cómo fue que llegaron esos profesores? ¿A través de qué programas y acciones? ¿Cuáles son sus experiencias de inserción en los nuevos espacios académicos?

Los participantes en el estudio forman parte del personal de tiempo completo (profesores investigadores o profesores, según el caso) en dos de las universidades públicas más grandes de la región latinoamericana, la Universidad Nacional Autónoma de México (UNAM) y la Universidad de São Paulo (USP). Para efectos de este documento, sólo se retomaron un total de ocho entrevistas ${ }^{2}$ de tipo semiestructuradas llevadas a cabo en los años 2011, para el caso mexicano y en el 2012, para un sondeo en el caso brasileño (IZQUIERDO, 2013). El objetivo del estudio fue conocer las experiencias de migración-inmigración de los científicos de la ex URSS. En este espacio, se aborda su inserción a los nuevos espacios académicos y se indaga, de manera exploratoria, la construcción de redes de investigación desde México y Brasil. Para acceder a las experiencias de los ocho

\footnotetext{
${ }^{2}$ Los sujetos entrevistados no son la totalidad o mayoría de los científicos de la ex URSS que trabajan en dichas universidades. Para el caso mexicano, se ubicaron a 144 científicos que llegaron a través del subprograma de Cátedras Patrimoniales y se establecieron en México, de los cuales 36 están laborando en la UNAM (Izquierdo, 2013). En el caso brasileño, no contamos por el momento con el número total de científicos de dicha región en ese país, una estimación hecha por un científico entrevistado en la Universidad de São Paulo, sugiere que existen "alrededor de 30" (Científico en Brasil, 2012-1) investigadores laborando en esa institución.
} 
científicos (cuatro de cada país), se utilizó el enfoque biográfico (BERTAUX, 1993), a través del relato de vida que, siguiendo al autor, son "relatos de experiencia".

El artículo consta de tres partes. En la primera, se presentó información sobre las oleadas migratorias de científicos de la ex URSS a diferentes partes del mundo. A continuación, se brindó un panorama general de esas migraciones hacia Latinoamérica, la cual es entendida en este documento, como una región que no sólo expulsa a su personal altamente calificado hacia el 'norte' sino que también atrae a profesionales extranjeros a sus espacios académicos del 'sur'. En la última parte, se expusieron algunas de las experiencias de migración, inmigración e inserción de los científicos de la ex Unión Soviética a los nuevos contextos académicos latinoamericanos, así como una exploración en la construcción de redes de investigación.

\section{MIGRACIONES DE CIENTÍFICOS DE LA EX URSS}

La migración de científicos de la ex URSS en los noventa cubrió casi todos los continentes (STREPETOVA, 1995). Algunos autores (PISKUNOV Y LEN'ŠIN, 1992; SHKOLNIKOV, 1994; KOUZNETSOVA, 1996; GOKHBERG Y NEKIPELOVA, 2002) afirmaron que esas corrientes migratorias se dirigieron principalmente a Estados Unidos, Israel y Alemania, pero también se incluyeron a Gran Bretaña, Australia, Canadá, Nueva Zelandia, Francia, Italia y Japón, así como los países del mediterráneo y escandinavos (UNESCO, 1994; OCDE, 1995; SIMANOVSKY ET. AL., 1996; RYBAKOVSKY Y RYAZANTSEV, 2005; LATOVA Y SAVINKOV, 2012), entre otros destinos.

Hasta el momento, no se ha podido cuantificar la migración total de científicos de aquella región, especialmente porque los datos que se pueden recabar sobre ellos son muy diferentes entre las fuentes y los parámetros utilizados para cuantificar el fenómeno. En los textos que se consultó, se encontró cifras referidas a científicos "rusos", pero no se especifica si en esa designación, se consideraron o no otras repúblicas que conformaron a la ex Unión Soviética. Por ejemplo, agencias de noticias, a nivel internacional, difundieron que arriba de 200,000 (PRAVDA 2005) o 440,000 dejaron Rusia (NOVAYA GAZETA, 2008); o bien que entre 500,000 y 800,000 (BBC NEWS, 2002) científicos, programadores de cómputo y especialistas rusos han emigrado. 
En otros casos, las fuentes muestran específicamente a los investigadores de Ucrania y de Rusia, brindando diferentes números, tanto por periodo como por sector: en 1990 Ucrania tenía 349,100 científicos y empleados en las universidades; 6,100 doctores en ciencia y 50,400 candidatos a doctor (UNESCO, 1994). Se estimó $^{3}$ que de 1988 a 1992 habían emigrado 219,500 científicos e ingenieros; tan sólo para el periodo de 1990 a 1992, 159 doctores en ciencia y candidatos a doctor habían emigrado de los institutos pertenecientes a la Academia de Ciencias de dicho país y 400 investigadores con grados universitarios se fueron a diferentes países para trabajar en empleos temporales, como a Israel, Estados Unidos, Francia, Gran Bretaña, países de Europa del Este, entre otros (Ibidem).

En el caso de Rusia, se afirmó que en 1991 tenía 878,482 científicos dedicados a la investigación y al desarrollo tecnológico y cuatro años más tarde contaba con 518,690 (GOKHBERG, 1998). En 1993 se identificó un total de 64,593 emigrantes rusos altamente calificados en países como Alemania, Israel, Estados Unidos, Grecia, Finlandia, Bulgaria, Canadá, Australia, Polonia, Suecia, Hungría, República Checa, Francia y otros (STREPETOVA, 1995). Entre el año 1989 y el 2000, de Rusia habían emigrado arriba de 20,000 académicos que estaban empleados como investigadores y asistentes de investigación y otros 30,000 especialistas que trabajan en el extranjero a través de contratos temporales (SADOVNICHY Y KOZLOV, 2005). Con respecto a los países y al número de científicos que han emigrado, se publicó que 30,000 científicos rusos trabajaban en Israel y Estados Unidos, más de 4,000 en Alemania, 600 en Francia y 95 en Corea (ORLOVA, ET. AL. 1994).

A toda esa heterogeneidad numérica habría que agregar, en primer lugar, la incertidumbre en el término de a quiénes considerar como profesionales altamente calificados (PARKHOMENKO, 2006). Además, se tendría que tomar en cuenta no sólo la cuantificación de la emigración permanente, sino también la llamada "fuga de cerebros interna", -es decir, los científicos que se quedaron en el país pero que cambiaron su campo laboral hacia las empresas bancarias, aéreas, de cómputo, de turismo y de servicios en general, así como aquellos que desarrollaron sus propios negocios- y la movilidad internacional que incluye los contratos temporales, las

\footnotetext{
${ }^{3}$ Aún en años recientes, especialistas afirmarían que es imposible definir con números reales la "fuga de cerebros" ucraniana (PARKHOMENKO, 2006).
} 
visitas cortas de investigación, de docencia y la formación académica especializada en el extranjero.

Habría que agregar también que los científicos que integran las áreas de las ciencias exactas y de las ingenierías, no fueron los únicos que emigraron. Se encontró reportes sobre científicos que se ubican en áreas de las ciencias sociales y humanidades, además de los países ya mencionados, aparecieron Austria y los Países Bajos como los lugares de destino (YELENEVSKAYA Y FIALKOVA, 2009). Otro grupo importante de migrantes fueron los artistas, como por ejemplo los músicos y los bailarines que han emigrado a Estados Unidos (RUESCHEMEYER, 1982) y a otras partes del mundo.

\section{MIGRACIONES DE CIENTÍFICOS DE LA EX URSS HACIA EL 'SUR'}

En este artículo se usó la denominación de 'sur' como una crítica a la idea unidireccional en la que se enmarcan los estudios sobre la migración altamente calificada en Latinoamérica (las Ilamadas migraciones 'sur-norte'). En el artículo, esa región aparece como un espacio de atracción en donde diversos grupos de científicos extranjeros también han arribado para trabajar y se han establecido de manera permanente. En ese sentido, es posible considerar la otra cara de la monedad del brain drain ("drenaje o pérdida de cerebros" latinoamericanos altamente calificados que migran al 'norte), a través del brain gain ("ganancia de cerebros" extranjeros altamente calificados inmigrantes en el 'sur') y de ello, se puede dar cuenta a través de la migración de científicos de la ex URSS a Latinoamérica.

En los noventa, se mencionó que habían ido a trabajar algunos científicos y técnicos nucleares de la ex URSS hacia Latinoamérica (KUDIMOV, 1992; MOODY, 1996). Más adelante, se reportó que la compañía de petróleo venezolana planeaba gastar 5 millones de dólares para contratar trabajadores altamente calificados de la ex URSS (ORLOVA, ET. AL. 1994) y que otros grupos "pequeños" de científicos e ingenieros de la ex URSS viajaron de manera temporal a Argentina y Uruguay (STREPETOVA, 1995). En la indagación que se realizó en Buenos Aires, no se encontró a algún científico trabajando en espacios universitarios, pero eso no significa que no existan, de hecho Argentina cuenta con una inmigración rusa y ucraniana muy importante, la cual suele concentrarse en el sector servicios. 
En general, se identificó a científicos de las ciencias exactas, aplicadas y de las ciencias sociales y humanidades de la ex URSS en México, Brasil, Colombia, Venezuela, Uruguay y Chile -en donde se intentó desarrollar un programa de atracción de científicos, parecido al subprograma mexicano pero, al final, no se concretó- y Cuba. En este último, especialmente continúan viviendo algunos técnicos, ingenieros y profesoras de ruso que nacieron en la ex URSS y que llegaron a dicho país para realizar "trabajo de cooperación científica" y de "asistencia técnica" en los setenta o porque se casaron con cubanos que fueron a estudiar a la Unión Soviética y arribaron al país en la década de los ochenta, en ambos casos se quedaron a vivir en Cuba de manera permanente.

\section{CIENTÍFICOS DE LA EX URSS EN MÉXICO Y EN BRASIL}

México y Brasil son países considerados en la categoría $A$ y $B$, según la clasificación del Banco Interamericano de Desarrollo (BID), en esa categoría también se incluyen otros países "grandes o medianos"4, como Argentina, Chile, Colombia, Perú y Venezuela. La línea que divide a éstos de los países "pequeños" que se ubican en la categoría C y D (Bahamas, Barbados, Belice, Bolivia, Costa Rica, Ecuador, El Salvador, Guatemala, Guyana, Honduras, Jamaica, Haití, Nicaragua, Panamá, Paraguay, República Dominicana, Surinam, Trinidad y Tobago, y Uruguay), se sitúa en torno a los 12 millones de habitantes y los 30.000 millones de dólares de su PNB (MAYORGA, 1997). Tan sólo para brindar una idea de la magnitud de los países de estudio, con respecto al número total de su población, en el 2011 Brasil tenía 194.49 millones de habitantes, mientras que México contaba con 114 millones (RICYT, 2011).

La atracción de científicos extranjeros hacia México y Brasil, en especial de los científicos de la ex URSS, no sólo estuvo basada en la inversión en ciencia y tecnología que cada gobierno ha hecho, o dejado de hacer en esos países- en el 2011 Brasil fue el país que más invirtió en toda la región, con un $1.21 \%$, muy al contrario de lo que sucedió en México, con el $0.46 \%$, por debajo de países como Argentina $(0.64 \%)$, Costa Rica $(0.48 \%)$ y rebasando a Chile $(0.45 \%)$, pero por muy poco (RICYT, 2013)-. Ese tipo de acción también obedeció a programas específicos que se implementaron para la atracción de científicos extranjeros, a través de una 
combinación de recursos económicos gubernamentales con financiamiento proveniente de organismos internacionales.

En el caso de México, en 1991 el gobierno federal implementó el Programa de Apoyo a la Ciencia en México (PACIME), que tuvo como objetivo fundamental "incrementar, de manera significativa, el desarrollo y difusión de la actividad científica del país, aumentando en número y en calidad la planta nacional de investigadores" (PACIME, 1991: 19). Para el desarrollo del Programa, el Banco Mundial otorgó un crédito al país por 150 millones de dólares y México aportó una contraparte equivalente (IBIDEM). El programa financió tres grandes subprogramas: proyectos de investigación, proyectos de infraestructura, repatriaciones y cátedras patrimoniales de excelencia, este último fue el subprograma a través del cual llegaron los científicos de la ex URSS al país. Según los datos encontrados, hubo aproximadamente 1000 registros de científicos extranjeros en dicho subprograma, de los cuales 261 provenían de la ex URSS; de ellos, continúan viviendo en el país 144 (IZQUIERDO, 2013). Si bien el subprograma de Cátedras Patrimoniales fue único en su tipo, se han desarrollado otros, quizá con menor capacidad, para la atracción de personal extranjero altamente calificado a México, en trabajos temporales y en movilidad académica.

Actualmente, el gobierno mexicano a través del Consejo Nacional de Ciencia y Tecnología (CONACyT), lanzó un programa llamado "Cátedras CONACyT para Jóvenes Investigadores", en el cual se prevé la contratación de 574 jóvenes doctores en todas las áreas del conocimiento. En dicho programa se resaltó la contratación no sólo de mexicanos, también de "extranjeros residentes legalmente en México" (CONACyT, 2014); con esa iniciativa se vuelve a abrir la puerta a la contratación permanente de científicos extranjeros en el país, la cual permanecía entrecerrada no porque no pudieran ser contratados sino porque no se había desarrollado un programa que surgiera en el seno del CONACyT con especificaciones y financiamiento para ello, desde la década de los noventa.

En el caso de Brasil, se informó que el Ministro de Educación brasileño declaró que ese país estaba listo para contratar a 10,000 científicos de la ex Unión Soviética en las universidades y centros de investigación en ese país (ORLOVA, ET. AL. 1994). Por el momento, no se tiene elementos para confirmar si se contrató esa

4 "Algunas regiones de países grandes (por ejemplo, nordeste de Brasil, sur de México) tienen situaciones de C\&T semejantes a las de países pequeños y más pobres" (MAYORGA, 1997). 
cantidad de científicos en Brasil, pero lo que sí ocurrió fue que varios grupos de personal altamente calificado de aquella región, se fueron a trabajar a ese país.

Los científicos entrevistados en Brasil, afirmaron que ellos no fueron reclutados a través de un programa o subprograma específico de atracción de personal altamente calificado, como en el caso mexicano, sino que más bien fueron invitados por sus colegas y amigos, tanto connacionales como brasileños para ir a trabajar a ese país. Sus contactos los construyeron a través de redes sociales y académicas y estas se fueron fortaleciendo con el tiempo y en eventos específicos, como congresos y seminarios internacionales, en los cuales los científicos participaron:

\begin{abstract}
No tuvimos un programa de ese tipo [se refiere al mexicano], simplemente no lo tuvimos, no era una política de gobierno o una acción o un programa, todo era por amistades, por invitaciones, que tú conoces a un colega y te invita "puedes venir a trabajar". Mis colegas brasileños me dijeron que habían perdido esa oportunidad, la oportunidad de hacer eso, que debieron haber hecho eso, perdieron la oportunidad de hacer algo estructurado así como en México, ese programa que me dice [Subprograma de Cátedras Patrimoniales], pero no, aquí no fue así, simplemente fueron contactos personales, por colegas que conocíamos de aquí y eso. Esa fue como la primera forma, luego, después, ya que estuvimos aquí, nosotros hicimos las invitaciones a nuestros colegas o conocidos de allá, hicimos las invitaciones nosotros, pero eso ya fue después, ya cuando estábamos establecidos aquí, a finales de los noventa más por la década del 2000 (Científico en Brasil, 2012-1).
\end{abstract}

Actualmente, Brasil cuenta con un programa muy importante llamado "Ciencia sin fronteras", en el cual se busca la atracción de científicos a ese país, a través de dos subprogramas: investigadores visitantes, dirigido a científicos de reconocido prestigio internacional y jóvenes talentos (MCTel, 2013), en ambos casos la puerta está abierta para la contratación de científicos extranjeros. Otra de las vías en que los científicos jóvenes extranjeros están llegando a Brasil (y a México) es a través de las estancias posdoctorales. En el caso de los científicos de la ex URSS ya establecidos en ese país, esa es una de las maneras en que ellos continúan el contacto y la línea de formación de jóvenes rusos y ucranianos y, a su vez, es una forma en que éstos jóvenes pueden ir abriéndose camino en el campo laboral latinoamericano.

Ya sea que los gobiernos hubieran puesto, o no, en marcha un programa particular para la atracción de los científicos de la ex Unión Soviética a Latinoamérica, las experiencias que a continuación se relataron, nos muestran que al final, estos científicos llegaron y se instalaron en los espacios académicos 
mexicanos y brasileños y que han hecho de esos espacios, lugares entrañables de vida para ellos y sus familias.

\title{
SALIDAS DE SUS PAÍSES DE ORIGEN
}

Para el caso de los científicos que viven y trabajan en México como para los que se encuentran en Brasil, los motivos de migración fueron similares; éstos se centraron fundamentalmente en razones económicas y en el desmoronamiento del aparato científico en la ex URSS, lo que les impidió desarrollar el trabajo para el cual habían sido formados. Cuestiones como la reducción del financiamiento a la ciencia, la caída de los salarios a los investigadores, el desmantelamiento de la infraestructura de los laboratorios e instituciones en educación superior y científicas, el declinamiento del prestigio de la labor intelectual, la falta de oportunidades para desarrollar el potencial científico, entre otros, fueron las causas que integraron la migración de los científicos (MOODY, 1996; IVAKHNYUK, 2006; GENOV, 2007). Un científico que trabaja en México, relató su experiencia de la siguiente manera:

\begin{abstract}
Se veía claramente que la imagen del científico se iba deteriorando. Mira, yo nací en una familia de científicos, mis dos abuelos eran científicos, uno, como comenté, era ingeniero aeroespacial y otro era matemático, [...]. Entonces, mis abuelos por ejemplo, yo me acuerdo cuando era niño, sí tenían un estatus muy elevado en la sociedad pero cuando yo llegué en los años de 1980 ya no era tan elevado, se iba deteriorando, así, yo creo que con el deterioro del Estado, así, que todo se venía abajo también. El reflejo de lo que representaba la ciencia, la ciencia refleja el estado del Estado, así, iba también deteriorándose poco a poco y en los años de 1990, yo ya no estaba en la Unión Soviética, yo estaba aquí pero, mis amigos y los periódicos decían que ser científico era ser vergonzoso porque no tenían nada de recursos, ni ganaban nada (Científico en México, 2011-1).
\end{abstract}

Según el relato anterior, el proceso de derrumbe del sistema científico inició en los ochenta, con "el deterioro del Estado", dicha tensión apareció claramente hasta la década de los noventa, fue en esa década cuando "ser científico era ser vergonzoso porque no tenían nada de recursos, ni ganaban nada". En todos los relatos se encontró que esos motivos concretaron la migración de los científicos porque fue hasta ese momento que ellos realizaron acciones específicas para poder salir, aunque desde la década de los ochenta ya existía cierta apertura para emigrar de aquella región. El poco financiamiento a la ciencia se hizo notar hasta llegar a la reducción de sus salarios o a la falta de pago de estos, "el sueldo de un jefe de laboratorio no supera actualmente [en 1994] los 36,000 rublos, que equivalen a unos 36 dólares. Y de los especialistas de rangos menores, mejor ni hablar" (GOGOL Y 
KUCHEROV, 1994: 88). Lo anterior también coincidió con la narración de los científicos que se entrevistaron en Brasil, además de los cambios sociales y políticos a raíz de la independencia de las repúblicas:

\begin{abstract}
Yo viví 4 años en Canadá, durante ese periodo la Unión Soviética colapsó, cuando yo regresé, era un país diferente. Yo regresé a Ucrania y ya era un país independiente, la mentalidad soviética había cambiado, se volvió más al estilo americano, el estilo de seguir el dinero, veías dólares y era la gran cosa, antes no veías dólares, no era así. [...]. Todo cambió por ejemplo, en la ciencia se vio como un cambio bueno para los más jóvenes porque podían cambiar, pero no para las personas viejas, ellos ya no podían cambiar las formas en las que habían vivido o trabajado antes y tampoco tenían oportunidad de hacer ese cambio porque todo fue muy rápido y para la generación mediana, la gente de en medio, ni jóvenes ni viejos, no digo que estábamos perdidos pero era como estar en el limbo, no sé, debías olvidarte de hacer lo que hacías y dedicarte a hacer algo más y ser bien flexible e integrarte al comercio o simplemente irte y mucha gente se fue, yo me fui (Científico en Brasil, 2012-3).
\end{abstract}

En plena crisis financiera, se ubicó a científicos que pusieron en marcha estrategias para poder "sobrevivir" o como uno de ellos lo refirió "todos buscábamos caminitos". Uno de los "caminitos" fue diversificar su ejercicio laboral, tanto en la ex Unión Soviética como fuera de ella. En un reporte realizado a principios de los noventa, se encontró que algunos científicos "iban a comerciar" sus servicios a empresas intermediarias que se dedicaban a la reventa de cualquier tipo de mercancía; otra manera fue participar en las "asociaciones científicas" haciendo alianzas con "hombres de negocios y empresarios, con fines prácticos. Algunos de esos profesionales, por ejemplo, venden programas de computadoras o dan servicios a las antenas parabólicas" (GOGOL Y KUCHEROV, 1994: 89).

En el caso de los científicos que tuvieron esa posibilidad, otro de los "caminitos" fue salir al extranjero durante ese periodo, esa fue también una manera de obtener recursos económicos y de salir de manera temporal a otros espacios académicos, continuar con su trabajo científico y de indagar posibilidades para encontrar un empleo permanente. Lo anterior, se señaló en el caso de un científico radicado en Brasil, que fue a trabajar a España por periodos cortos hasta que conoció a unos colegas brasileños que también se encontraban en la misma universidad española que él y lo invitaron a trabajar a dicho país.

\title{
LLEGADAS Y ESTANCIAS EN LOS NUEVOS ESPACIOS ACADÉMICOS
}

Los científicos contaban con poca información sobre los países a donde iban a emigrar. Conocían que tanto México como Brasil tenían una diversidad de flora y que 
también contaban con artistas y escritores importantes. Excepto por el científico que había trabajado en España y aprendió español y que luego se fue a Brasil, ninguno de los investigadores en este estudio sabía español/portugués antes de llegar a México y a Brasil.

Las primeras impresiones que los científicos relataron sobre su llegada a Latinoamérica fueron en lo cultural. Las diferencias entre las formas de vestir, el tipo de comida y el idioma, fueron elementos del repertorio cultural mexicano y brasileño que les impactó en sus primeros días de haber llegado a dichos países. El choque con los dos primeros elementos -las formas de vestir y la comida- tuvieron que ver con las propias maneras en que los científicos concebían el vestido y el alimento en su propia cultura. El tercer elemento -el idioma- tuvo que ver no sólo con su desconocimiento del español/portugués y con ello, la imposibilidad de poder comunicarse con los otros, sino también con la sensación del silencio súbito, "me quedé como mudo" -reconoció un científico en México- para poder expresar sus emociones.

Al no saber el idioma, difícilmente los científicos podían expresar esas emociones que emergían de sus experiencias visuales, olfativas y gustativas en el mismo momento en que estas ocurrían y en el mismo idioma que las provocaba. Ese tipo de experiencias, también han sido reportadas en otros estudios (AL-HAJ 2002; KHEIMETS Y EPSTEIN, 2001), en los que se incluye a personal altamente calificado de la ex Unión Soviética en Israel, para saber el grado de adaptación, de integración y de reconstrucción de identidades en la sociedad de acogida.

En los casos que se presentan, no todas las impresiones estuvieron centradas en las experiencias de los científicos con relación a las sociedades de llegada, también aparecieron primeras impresiones sobre los espacios de trabajo, de sus colegas mexicanos/brasileños y del ambiente laboral. En las siguientes citas, se puede apreciar que las primeras impresiones fueron de "sorpresa", no imaginaban que en México y en Brasil se pudiera tener "tan buenas condiciones de trabajo" en comparación con sus países, en la década de los noventa:

Cuando yo vine aquí yo estuve sorprendido de que tuvieran tan buena librería [biblioteca], podía acceder a todas las revistas que yo quisiera porque en los últimos años en Rusia, nuestro acceso a los papers internacionales no era posible, no teníamos dinero para hacer esa inversión. En segundo lugar, mi impresión fue que tenían un buen centro de computación en comparación con los últimos años en mi laboratorio. Mi tercera impresión fue que el Instituto de Física tenía muchas conexiones y buenos científicos de buen nivel (Científico en México, 2011-4). 


\begin{abstract}
En Brasil llegué a trabajar a un muy buen instituto. Yo platicaba con mis colegas que nosotros somos afortunados, tenemos una gran institución, tenemos a 150 especialistas en ciencia pura en este instituto. [...]. Otra cosa buena en Brasil es que como extranjero tú eres absolutamente bienvenido, bueno, no absolutamente, en cada país hay gente que no se siente muy cómodo de que extranjeros vengan y se queden, pero aquí es muy positivo, nos sentimos muy bienvenidos y los brasileños son agradables con nosotros (Científica en Brasil, 2012-2).
\end{abstract}

Las impresiones en relación con sus espacios laborales y la infraestructura en México y en Brasil, generalmente tuvieron el referente de la comparación con lo que ellos dejaron de tener en su país al momento de emigrar: la "oportunidad" de encontrar un espacio laboral que les permitiera desarrollar sus capacidades académicas para las cuales habían sido formados. Sus narraciones también aludieron a las diferencias con respecto a las formas de trabajo científico en los nuevos contextos académicos a los que se incorporaron. Por ejemplo, los científicos en México encontraron que no había "técnicos especializados", que no había financiamiento directo a sus proyectos, ellos tenían que competir para obtener recursos. En el caso de los científicos en Brasil, uno de ellos externó su dificultad en su nuevo contexto universitario en términos de formación académica, "mi experiencia aquí no fue fácil porque nosotros tenemos otra cultura, otro entrenamiento, venimos de distintas escuelas en la ciencia, eso nos hace diferentes con los brasileños". Si bien los científicos manifestaron haber tenido tensiones culturales entre sus países de origen y los países de llegada, las "oportunidades" laborales que encontraron tanto en México como en Brasil fueron muy importantes para establecerse en esta región:

Cuando me invitaron, yo entendí "sí me van a valorar", eso fue una atracción "me van a valorar porque no estoy buscando, ellos me buscan a mí". Eso fue lo principal, que te valoran, ya no tienes que demostrar, en nuestro país hay un dicho "no tienes que demostrar que tú no eres camello". Es decir, si tú vas a otro país te dicen "demuéstrame quién eres, tus papeles". Yo soy un científico ¿por qué voy a demostrar? no debo de demostrar que no soy camello ¿No? [...] (Científico en México, 2011-3).

Yo llegué aquí sin dinero, perdí todo en Rusia. Después de cuarenta y ocho años de trabajo, vine a Brasil sin nada, cero ¿puedes imaginarlo? [...]. No, yo no lo elegí. Yo no tuve opciones. Brasil me ofreció una posición, entonces yo vine. A mí me conoció un científico brasileño porque yo era muy conocido en el mundo, ellos me conocían, ellos sabían de mi por mi currículum, mi currículum no era tan malo [ríe], yo tenía libros publicados, los físicos en mi área, me conocían. Ellos me invitaron desde un principio para una posición permanente, yo no acepté inmediatamente, yo vine y conocí a la gente, la universidad, la situación que me ofrecían y después yo decidí quedarme. Yo también fui invitado a obtener una posición en México. Fui allá, pero no me gustó, sentí que Brasil era más suave que México. Aquí la gente es más amigable que en México, la gente es más agradable aquí, 
las condiciones de trabajo son mejores, todo era mejor, así que decidí no aceptar en México (Científico en Brasil, 2012-4).

Los científicos provenían de una cultura en la cual, la ciencia tuvo uno de los más importantes papeles en el desarrollo de las ex repúblicas en donde habían nacido. Pero a partir de los sesenta-setenta, ese papel se fue desdibujando hasta borrar la imagen de la ciencia y de los científicos como elementos fundamentales en el sostenimiento, no sólo económico también identitario de aquella región. En los noventa, los científicos que alguna vez gozaron de "respeto" y "admiración" de la sociedad, ya no eran necesarios en sus países de origen pero sí fueron requeridos en México y en Brasil. En ambas citas se pudo apreciar que los científicos se sintieron valorados por su trabajo, ese fue uno de los motivos del por qué decidieron establecerse en los espacios de investigación y docencia latinoamericanos.

\section{TRABAJO DOCENTE Y REDES DE INVESTIGACIÓN}

Una vez que los científicos de la ex URSS se establecieron en las instituciones universitarias, resaltaron dos actividades principales: la docencia y la investigación. En ambos casos, sus prácticas académicas cambiaron al trasladarse a los nuevos contextos académicos en México y en Brasil pero encontré que los científicos intentaron mantener algunas de ellas. A continuación se muestra un ejemplo de ello, con respecto a su trabajo docente:

\footnotetext{
Traté de hacer algo en educación sistemática para que el estudiante no memorice, [...], ahí la ventaja del sistema soviético fue que no nos enseñaban a memorizar, vamos, Física Cuántica ¿Qué vas a memorizar? [...], como decían nuestros profesores, no es la suma de conocimiento memorizado sino es la actitud hacia el conocimiento, eso sí les estoy enseñando. [...], estos chicos, no digo que van a revolucionar la ciencia mundial pero esa actitud estoy enseñando ¿no? "Mira, piensa, critícalo, mira, hicieron esto ¿No tienen nada? Critícalo ¿Por qué hicieron eso?" (Científico en México, 2011-2).
}

Al igual que en su quehacer docente, en las prácticas de investigación de los científicos también identifiqué cambios pero, mientras en la primera se hizo un esfuerzo por implementar las maneras en las que ellos fueron formados por sus maestros, en la segunda, el esfuerzo se centró en el rescate de sus propias experiencias laborales en la ex URSS -para el caso de quienes las tuvieron-. Los cambios estuvieron relacionados con las condiciones laborales de las instituciones en las que ellos se incorporaron, entre ellos se mencionó la posibilidad de poder viajar para presentar sus investigaciones en congresos e iniciar colaboraciones de 
trabajo en diferentes partes del mundo con la comunidad científica a nivel internacional:

\begin{abstract}
Aquí obtuve muchos contactos, más contactos que antes que yo estaba en Rusia. Mi primer año en México yo viajé 4 o 5 veces por conferencias, fui a Estados Unidos, Inglaterra, Italia. Y en este caso yo trabajaba en Inglaterra y allá fui dos veces por un mes con X, él es miembro de la Royal Society y nosotros tuvimos muy buena colaboración (Científico en México, 2011-4).
\end{abstract}

Me gustó la universidad que es muy buena, tiene muy buena especialidad para hacer investigación, para invitar a otros científicos, para desarrollar proyectos es muy buena. Yo creo que es una de las mejores universidades para desarrollar proyectos. En España por ejemplo, era bueno pero, yo siempre me sentí como un huésped, entonces si yo quería invitar alguien, no tenía esa, tenía libertad en mis investigaciones y de hacer lo que quería en mis investigaciones pero, viajar e invitar, no, en ese sentido, no. Y aquí es diferente, aquí puedo invitar y puedo viajar (Científico en Brasil, 2012-1).

Además de los viajes y las colaboraciones internacionales, algunos de los científicos tuvieron que cambiar sus temas investigación -más no la disciplinaporque fue una solicitud que les hicieron al llegar o porque ellos tomaron dicha decisión previendo que las condiciones en los centros de trabajo a donde habían llegado eran más favorables para, por ejemplo, trabajar en temas aplicados o teóricos, según la situación en la que se encontró cada uno de los participantes, en especial esto lo encontré en el caso de los científicos en México.

En su trabajo de investigación, un aspecto que resaltó fue la construcción de redes que los científicos empezaron a gestar desde su llegada a México y a Brasil, y algunos de ellos, desde antes. Si bien los incipientes nexos que los científicos tenían al momento de sus incorporaciones no se pueden catalogar como una red de investigación en sí misma, en el estricto sentido del concepto que algunos autores manejan (ARELLANO Y JENSEN, 2006), aquí se usó esa idea para mostrar que desde su arribo a esta región, algunos de ellos ya contaban con ciertos vínculos con la comunidad académica mexicana y brasileña.

Esos lazos se fueron tejiendo a lo largo de los años hasta lograr lo que ahora se puede catalogar como una robusta red de investigación, con varios nodos y lazos, no sólo en los países de llegada, también en colaboración en otras partes del mundo, en la que se destaca la comunicación formal a través de los artículos publicados (LIBERMAN Y WOLF, 1990). Sin embargo, una diferencia que se encontró entre los científicos de la ex URSS en México y en Brasil, es que los primeros no suelen construir redes de investigación con sus colegas que radican en sus países de origen: 


\begin{abstract}
Ya no tengo contactos con ellos. Yo cuando, por ejemplo, cuando tengo un congreso en Alemania o cerca de Rusia, yo visito Rusia, los visito con un reporte o doy dos reportes en algún seminario, solamente hago eso, pero no tengo colaboración o trabajo en común con ellos, ni publicaciones. Desde que llegué a México no hice contactos, como mi tema cambió, ya eso no se pudo dar, después entré a la vida académica aquí, con otros colaboradores y estudiantes y ya eso no fue posible (Científico en México, 2011-4).
\end{abstract}

En el caso de los científicos en Brasil, si bien dijeron tener contactos y algunas publicaciones con sus colegas que radican en sus países de origen, ese vínculo se ha ido debilitando. En ambos casos, los científicos mantienen nexos importantes con sus colegas provenientes de la ex URSS pero éstos se encuentran, como ellos, en el extranjero ¿Por qué la reticencia a construir redes con sus connacionales en sus países de origen? ¿Cuáles han sido las razones del debilitamiento de los vínculos que tenían con sus colegas en sus países de origen, cuando llegaron a esta región?

Hasta ahora, los motivos que se han ubicado son tres. El primero tiene que ver con el sentimiento de culpa que algunos científicos expresaron por haber salido del país, "los más patriotas fueron los que se quedaron", "a veces te ven mal, que fuiste un traidor por salir ¿no?”. Quizá, una manera en que los científicos evitaron enfrentarse a ese tipo de sentimientos, fue cortando el vínculo desde el principio. Otra razón fue que los temas de investigación de algunos científicos inmigrantes en Latinoamérica han cambiado y eso ya no les permite continuar con el lazo que habían construido cuando trabajaban con sus colegas que viven en la ex URSS. Y, finalmente, se encontró que para ciertos científicos -especialmente para los más jóvenes-, su motivación tuvo un sentido pragmático: ha resultado más conveniente vincularse con otros colegas de su mismo país de nacimiento que viven en Estados Unidos por ejemplo, que con sus pares que radican en sus países de origen, por la cuestión del financiamiento al que pueden acceder desde dichos espacios y por la cercanía geográfica en la que actualmente se ubican.

\title{
CONSIDERAÇÕES FINAIS
}

En las exploraciones sobre la inmigración de científicos de la ex Unión Soviética en Latinoamérica, se ubicó a México y a Brasil como dos de los 'polos' principales hacia donde se han dirigido y establecido estos grupos. Los itinerarios que han recorrido para llegar a ambos países fueron diferentes y también las formas en las que se incorporaron a los espacios académicos laborales. Mientras que en México se implementó un subprograma específico de atracción de científicos extranjeros, en 
Brasil, la llegada de éstos fue a través de vínculos con colegas, pero mientras el primero tuvo que solicitar un préstamo al Banco Mundial para llevarlo a acabo, el segundo no acudió a dicha práctica, de hecho el gobierno brasileño es el que más ha invertido en el desarrollo en ciencia y tecnología, destacándose en toda la región.

No todo fue diferencia. En ambos casos, los científicos coincidieron en que los motivos de migración de sus países de origen fueron principalmente económicos. La crisis económica que se venía arrastrando desde décadas antes a su salida de aquella región, impactó en el sistema científico tecnológico de tal manera que a su llegada a México y a Brasil, los científicos reconocieron tener mejores "oportunidades" para su desempeño académico. Una vez en Latinoamérica, su trabajo en investigación y docencia tuvo transformaciones, la diferencia más importante que se pudo identificar fue que mientras en la primera hicieron un esfuerzo por implementar las maneras en las que fueron formados por sus maestros, en la segunda, ese esfuerzo se volcó en el rescate de sus propias experiencias laborales en la ex Unión Soviética.

En la exploración sobre su trabajo científico, uno de los indicios que apareció fue la cuestión de las redes de investigación que han construido desde sus actuales espacios académicos. Se ubicó de manera preliminar que los científicos, tanto en México como en Brasil, en especial en el primer caso, no suelen mantener vínculos con sus colegas connacionales que viven en sus países de origen. En el caso de los que se encuentran en el segundo país, ese nexo se ha debilitado. Hasta ahora existen tres motivos que parecen explicar esos comportamientos, sin embargo aún falta por estudiar a fondo este tema como para poder brindar conclusiones. Si acaso lo que se puede hacer en este espacio es compartir algunas aperturas delineadas por preguntas: ¿Cómo se relacionan las comunidades científicas inmigrantes desde los espacios académicos latinoamericanos? ¿A través de qué formas de colaboración e intercambios? ¿Qué acciones o estrategias ponen en marcha los científicos para lograrlo? El artículo que se presentó, es un primer acercamiento que en el futuro pretende contribuir en ese sentido.

\section{REFERÊNCIAS}

AL-HAJ, M. Identity patterns among immigrants from the Former Soviet Union in Israel: assimilation vs. ethnic formation. International Migration, vol. 40, n. 2, pp. 49-69. 2002. 
ARELLANO, A. Y H. JENSEN. Mapeando las redes de investigación en Ciencias Básicas en la Universidad de Costa Rica. Convergencia, vol. 13, n. 42, pp. 181213. 2006.

BBC NEWS Russian brain drain tops half a million <Disponible en http://news.bbc.co.uk/2/hi/europe/2055571.stm>, 2002.

BERTAUX, D. La perspectiva biográfica: validez metodológica y potencialidades. José Miguel Marinas y Cristina Santamarina (Eds.) La historia Oral: métodos y experiencias. Debate. 1993.

CONSEJO NACIONAL DE CIENCIA Y TECNOLOGÍA. Cátedras CONACYT para Jóvenes Investigadores. México Distrito Federal: CONACyT. 2014.

GENOV, NIKOLAI. Brain-drain from Eastern Europe? What we know about and what not? Workshop EU enlargement and the labor markets. Bonn, September n. 78, pp. 1-13. 2007.

GOGOL, N. Y V. KUCHEROV. Rusia: la ciencia está mal pero... los científicos no se apresuran a emigrar. Ciencia y Desarrollo XX, n. 115, pp. 88-90. 1994.

GOKHBERG, L. La comunidad de Estados Independientes, en UNESCO, Informe mundial sobre la ciencia. Madrid: Santillana. 1998.

GOKHBERG, L. Y E. NEKIPELOVA. International Migrations of Scientists and Engineers in Russia. International Mobility of the Highly Skilled, OCDE. 2002.

IVAKHNYUK, I. Brain Drain from Russia: in Search for a Solution. Reports and Analyses. Center for International Relations n. 15, pp. 1-14. 2006.

IZQUIERDO, I. Tensiones, distensiones y estrategias identitarias. El caso de los científicos de la ex URSS inmigrantes en México en los noventa. Tesis de doctorado. México Distrito Federal: Universidad Nacional Autónoma de México. 2013.

KHEIMETS, N. Y A. EPSTEIN. English as a central component of success in the profesional and social integration of scientists from the Former Soviet Union in Israel. Lenguage in Society, vol. 30, n. 2, pp. 187-215. 2001.

KOUZNETSOVA, T. Brain Drain: Problem of Contract Migration in Russia <Disponible en http://horizon.documentation.ird.fr>, 1996.

KUDIMOV, Y. Russian physicists working in Latin America. Novoye Vremya, $\mathrm{n}$. 9, pp. 26-27. 1992.

LATOVA, N. Y V. SAVINKOV. The influence of Academic migration on the intellectual potential of Russia. European Journal of Education, vol. 47, n. 1, pp. 64-76. 2012.

LIBERMAN, S. Y B. WOLF. Las redes de comunicación científica. México Distrito Federal: UNAM. 1990. 
LOZANO, F. Y L. GANDINI. Migrantes calificados de América Latina y el Caribe ¿Capacidades desaprovechadas? México Distrito Federal: UNAM. 2011.

MAYORGA, R. Cerrando la brecha. Washington, D.C.: Banco Interamericano de Desarrollo. 1997.

MINISTERIO DE CIENCIA, TECNOLOGÍA E INNOVACIÓN. <Disponible en http://www.cienciasemfronteiras.gov.br/web/csf/pesquisador-visitante-especial>, 2013.

MOODY, A. Reexamining brain drain from the Former Soviet Union. The Nonproliferation Review. Spring-Summer, pp. 92-97. 1996.

NOVAYA GAZETA. People left Rusia. Novaya Gazeta, n.46, p.1. 2008.

OCDE. The Measurement of Scientific and Technological Activities Manual on the Measurement of Human Resources Devoted to S \& T Canberra Manual. París: OCDE. 1995.

ORLOVA, I., Y. STRELTSOVA Y E. SKVORTSOVA. Contemporary Migration Processes in Rusia. Refuge, vol. 14, no. 2, pp.1-17. 1994.

PARKHOMENKO, N. Brain Drain from Ukraine: current situation and future challenges. Center for Peace, Conversion and Foreign Policy of Ukraine. 2006.

PISKUNOV, D. Y V. LEN'ŠIN. The choice facing Russian science: partnership or brain drain. Economic Policy in Transitional economies, vol.2, n. 3, pp. 123-133. 1992.

PRAVDA. Immigration of scientists causes up to $\$ 30$ billion of damage to Russia <Disponible en http://english.pravda.ru/main/18/88/351/16590_scientists.html>, 2005 .

PROGRAMA DE APOYO A LA CIENCIA EN MÉXICO PACIME. México Distrito Federal: CONACyT. 1991.

RED DE INDICADORES DE CIENCIA Y TECNOLOGÍA IBEROAMERICANA E INTERAMERICANA (RICYT). Indicadores comparativos de población, sede de la coordinación: Centro de Estudios sobre Ciencia, Desarrollo y Educación Superior. Madrid: Organización de Estados Iberoamericanos (OEI). 2011.

RED DE INDICADORES DE CIENCIA Y TECNOLOGÍA IBEROAMERICANA E INTERAMERICANA (RICYT) El estado de la ciencia 2013, sede de la coordinación: Centro de Estudios sobre Ciencia, Desarrollo y Educación Superior. Madrid: Organización de Estados Iberoamericanos (OEI). 2013.

RUESCHEMEYER, M. The emigration Experience of Soviets Artists in the United States. Canadian Slavonic Papers, vol. 24, n. 3, pp. 261-272. 1982. 
RYBAKOVSKY, L. Y S. RYAZANTSEV. International migration in the Russian federation. New York: United Nations (UN). Department of Economic and Social Affairs. 2005.

SADOVNICHY, V. Y B. KOZLOV. The Russian Federation. Science Report, París: UNESCO. 2005.

SHKOLNIKOV, V. Scientific bodies in motion. The domestic and international consequences of the current and emergent Brain Drain from the Former USSR, Santa Monica: RAND. 1994.

SIMANOVSKY, S., M. STREPETOVA Y Y. NAIDO. Brain drain from Russia: problems, prospects, and ways of regulation. Ney York: Nova Science Publishers. 1996.

STREPETOVA, M. The brain drain in Russia. Budapest: Academy of Sciences. 1995.

UNESCO. Brain drain issues in Europe: Cases of Russia and Ukraine, Regional office for Science and Technology for Europe (ROSTE), n. 18, p. 210. 1994.

YELENEVSKAYA, M. Y L. FIALKOVA. The case of former Soviet scientists. Eliezer Ben-Rafael y Yitzhak Sternberg (Eds.) Transnationalism: Diasporas and the advent of new (Dis)order. Brill. 2009. 